\title{
Irrigación e Inervación de la Articulación Temporomandibular. Una Revisión de la Literatura
}

\author{
Irrigation and Innervation of the Temporomandibular Joint. A Literature Review
}

\author{
Ramón Fuentes*; Nicolás Ernesto Ottone ${ }^{*, * *}$; Diego Saravia*** \& Cristina Bucchi*
}

FUENTES, R.; OTTONE N. E.; SARAVIA, D. \& BUCCHI, C. Irrigación e inervación de la articulación temporomandibular. Una revisión de la literatura. Int. J.Morphol., 34(3):1024-1033, 2016.

RESUMEN: El conocimiento de los nervios aferentes, de la irrigación vascular y del drenaje venoso comprometido en la articulación temporomandibular es relevante para entender la fisiología del sistema estomatognático. El objetivo de este estudio fue identificar los vasos sanguíneos y los nervios involucrados en la irrigación e inervación de la ATM, y si los términos utilizados en la literatura científica respetan lo establecido en Terminologia Anatomica Internacional (TAI). Se realizó una revisión de la literatura en la que se incluyeron 10 libros y 11 artículos científicos. La mayoría de los autores señalaron que la irrigación de la ATM se lleva a cabo por ramas de la arteria temporal superficial y ramas de la arteria maxilar, que a su vez son ramas de la arteria carótida externa. El drenaje venoso estaría dado por las venas temporales superficiales y por el plexo pterigoideo. La inervación sensitiva provenía del nervio mandibular, ramo del $\mathrm{V}$ par craneal y sus ramos auriculotemporal, maseterino y los nervios temporales profundos. Los autores concuerdan en los principales vasos y ramos nerviosos que dan irrigación e inervación a la ATM, aunque también se mencionan otros vasos y nervios menos descritos. El drenaje venoso de la ATM se encuentra escasamente descrito en la literatura.

PALABRAS CLAVE: Articulación temporomandibular; Irrigación; Inervación; Drenaje venoso.

\section{INTRODUCCIÓN}

La articulación temporomandibular (ATM) es una articulación bicondilar sinovial de tipo gínglimo artroidal, que articula el cóndilo mandibular con el hueso temporal (Rubiano, 2005; Vasconcellos et al., 2007). La ATM tiene un componente óseo, muscular, ligamentoso y articular, los cuales han sido ampliamente estudiados en el ser humano. Sin embargo, las relaciones anatómicas de la neurovasculatura son usualmente descritas de manera genérica y superficial (Maciel, 2003).

El conocimiento de los nervios aferentes, de la irrigación vascular y del drenaje venoso comprometidos en la articulación temporomandibular es relevante para entender la fisiología y patología del sistema estomatognático. Adicionalmente, la arquitectura vascular afecta el planeamiento quirúrgico, en donde los disturbios vasculares causados a consecuencia de procedimientos quirúrgicos erróneos pueden tener secuelas serias (Sicher \& Dubrul, 1991).
La irrigación sanguínea da nutrición a la ATM y a los tejidos circundantes e influencia su crecimiento y desarrollo, pero también es un peligro potencial como vía de propagación de infecciones de origen dental, a través de los canales anastomóticos del plexo pterigoideo (Ebright et al., 2001; Colbert et al., 2011). La irrigación de la ATM está compuesta principalmente por ramas de la arteria temporal superficial y de la arteria maxilar (Maciel), aunque varios autores mencionan otros vasos sanguíneos, menos descritos (Velayos \& Santana, 2001; Sicher \& Dubrul). El drenaje venoso en tanto lo otorga mayoritariamente el plexo pterigoideo (Velayos \& Santana).

La inervación sensitiva está a cargo de los nervios auriculotemporal y masetérino, ramos del nervio mandibular, ramo del quinto par craneal (Velayos \& Santana; Sicher \& Dubrul) y de otros ramos nerviosos adicionales a los tradicionalmente descritos (Maciel).

\footnotetext{
* CICO - Centro de Investigación en Ciencias Odontológicas, Facultad de Odontología, Universidad de La Frontera, Temuco, Chile.

*** Programa de Doctorado en Ciencias Morfológicas, Facultad de Medicina, Universidad de La Frontera, Temuco, Chile.

**** Universidad Adventista de Chile, Chillán, Chile.
} 
El objetivo de este estudio fue establecer los vasos sanguíneos y los nervios involucrados en la irrigación e inervación de la ATM, a través de la revisión de artículos científicos y libros de anatomía. Adicionalmente, se evalúa si los términos utilizados por la literatura para referirse a los componentes nerviosos y vasculares de la ATM cumplen con lo establecido por la Terminologia Anatomica Internacional (TAI).

\section{MATERIAL Y MÉTODO}

Estrategia de búsqueda: La búsqueda electrónica sistemática de artículos científicos se realizó en las bases de dato MEDLINE, SciELO, Scopus, y ScienceDirect. La última búsqueda fue realizada en abril, 2015. Se utilizaron los términos de búsqueda: "blood supply", vascularization, "venous drainage", innervation, "neural elements", "nerve supply", anatomy, "temporomandibular joint" y TMJ y se combinaron con los términos boleanos AND y OR.

Adicionalmente, se incluyeron todos los libros de anatomía pertenecientes a una colección particular, escritos en idioma español, portugués e inglés, que trataban sobre la ATM y que contuvieran información referente a su irrigación e inervación.

Criterios de inclusión y exclusión de los artículos: Se incluyeron:

-Estudios que se orientaran a la descripción de la irrigación e inervación de la articulación temporomandibular. -Se incluyeron sólo estudios realizados en humanos, sin alteraciones o patologías.

-Estudios realizados en material cadavérico y/o imagenológico

-Artículo en idiomas español, inglés o portugués.

-Artículos de texto completo.

-No se aplicó restricción por fecha de publicación ni tamaño de muestra.

-Se excluyeron revisiones, cartas al editor y artículos técnicos.

Metodología de revisión: La búsqueda electrónica se realizó en dos oportunidades por un único investigador. La primera selección se llevó a cabo leyendo los títulos y/o resúmenes de los artículos otorgados por la búsqueda y se evaluó su atingencia al tema. Posterior a la primera selección de artículos, se analizó el texto completo y se verificó su cumplimiento con los criterios de inclusión. Finalmente, se realizó una búsqueda manual de la lista de referencias en los artículos incluidos.
Extracción de datos: La extracción de los datos fue realizada por un único investigador y la información obtenida fue ingresada en una ficha de recolección de datos. Los datos extraídos fueron los referentes a la descripción anatómica de las arterias, venas y nervios que dan irrigación e inervación a los componentes de la ATM. Adicionalmente, se compararon los términos para referirse a los vasos sanguíneos y nervios de la ATM, con los términos establecidos por la TAI en su versión latín, inglés y español (2001)

\section{RESULTADOS}

Un total de diez libros fueron incluidos, cinco correspondientes a textos de anatomía general o anatomía aplicada y cinco a especialidades odontológicas. Siete libros fueron publicados en idioma español y tres en portugués.

La estrategia de búsqueda en las bases de datos encontró 466 artículos referentes al tema. Diez cumplieron los criterios de inclusión y se incluyó un artículo más, encontrado a través de la búsqueda manual.

De los artículos científicos incluidos, nueve fueron realizados en material cadavérico y dos en pacientes sometidos a resonancia magnética con medio de contraste. Ocho artículos abordaron toda la vascularización o inervación comprometida en la ATM y tres se orientaron a la descripción de una arteria o nervio en particular. Ningún artículo describió el drenaje venoso de la ATM, por lo que la información contenida en este artículo referente a este tema, proviene enteramente de los textos analizados.

En la Tabla I se presenta el resumen de las arterias que dan irrigación a la ATM, según autor. En las Figuras 1 y 2 se observan las arterias comprometidas en la irrigación de la ATM y en la Figura 4 el drenaje venoso. En la Tabla II se presenta el resumen de los nervios que dan inervación sensitiva a la ATM, según autor. En la Figura 5 y 6 se esquematizan los nervios comprometidos en la inervación sensitiva de la ATM. Adicionalmente, en la Figura 3 se esquematizan las relaciones anatómicas de las arterias y nervios comprometidos en la irrigación e inervación de la ATM.

La mayoría de los autores utiliza los términos establecidos por la TAI para referirse a los nervios y vasos sanguíneos de la ATM. Sin embargo, existen algunas discrepancias observadas en la Tabla III. Las mayores diferencias con la TAI se refieren al nervio maseterino (término TAI), que es mencionado por varios autores como nervio masetérico (Velayos \& Santana; Maciel; Pertes \& Gross), 
Tabla I.- Resumen de las arterias que dan irrigación a la ATM, según autor. Se observa que prácticamente la totalidad de los autores señalan a la arteria maxilar y a la arteria temporal superficial como las responsables de la irrigación de la articulación.

ARTERIA CARÓTIDA EXTERNA

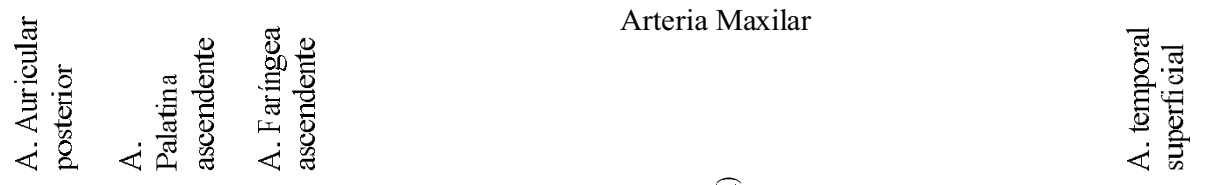

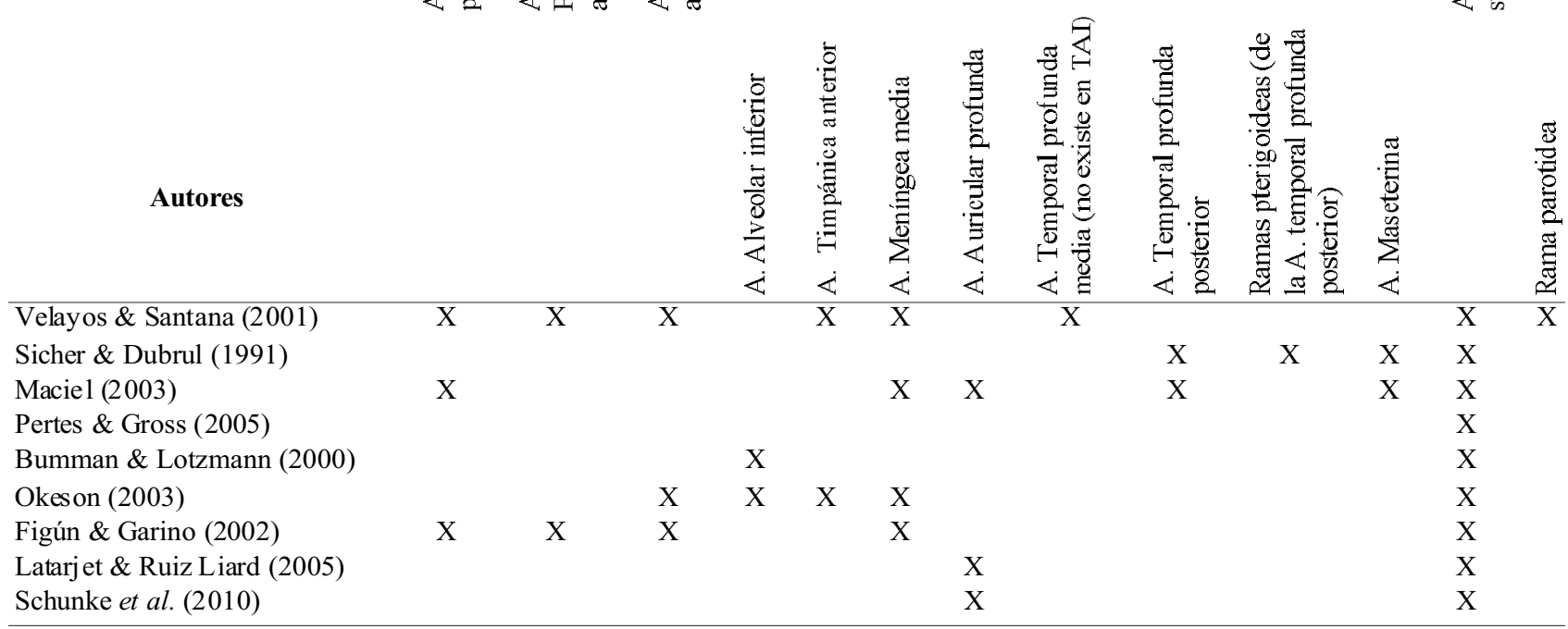

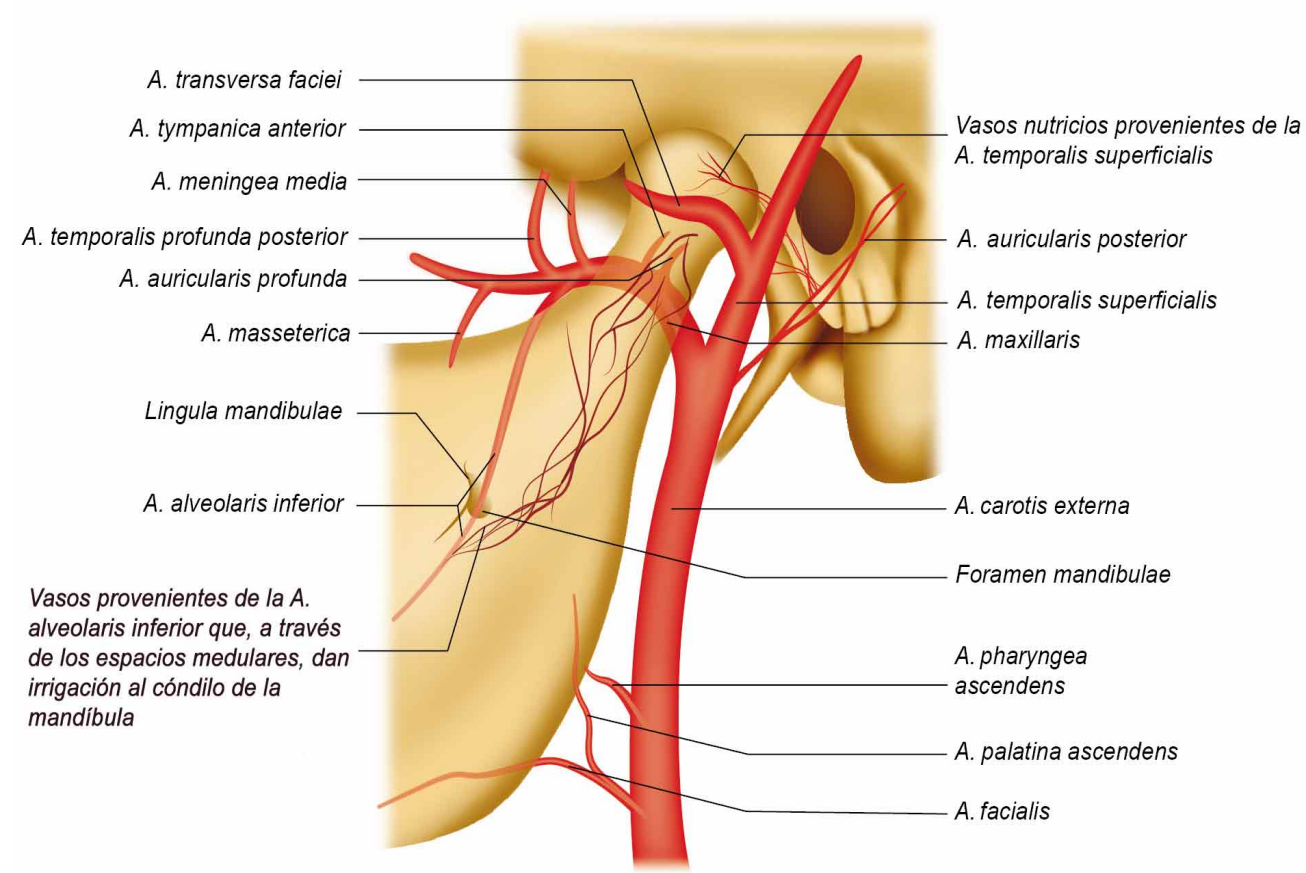

Fig.1. Vista lateral del cráneo. Esquema de todas las arterias comprometidas en la irrigación de la ATM.

posiblemente porque en latín e inglés este nervio es nombrado por la TAI como $N$. massetericus y masseteric nerve, respectivamente. También hay diferencias con respecto a los nervios temporales profundos que son nombrados por la mayoría de los autores en singular (nervio temporal profundo) (Sicher \& Dubrull; Pertes \& Gross; Okeson; Martínez-Ross, 1992). 
Tabla II. Resumen de los nervios que dan inervación sensitiva a la ATM, según autor. La totalidad de los autores señalan al nervio auriculotemporal y al nervio maseterino, ramos del nervio mandibular, como los responsables de la inervación de la articulación.

\begin{tabular}{|c|c|c|c|c|}
\hline \multirow[b]{3}{*}{ Velayos \& Santana } & \multicolumn{4}{|c|}{ Nervio mandibular } \\
\hline & N. auriculotemporal & N. Maseterino & Nn. temporales profundos & N. del músculo pterigoideo later al \\
\hline & - & - & & \\
\hline Sicher \& Dubrul & - & - & - & \\
\hline Maciel & - & - & - & - \\
\hline Pertes \& Gross & - & - & - & \\
\hline Bumman \& Lotzmann & - & - & - & \\
\hline Okeson & - & - & - & \\
\hline Martinez-Ross & - & - & - & \\
\hline Figún \& Garino & - & - & & \\
\hline Schunke et al. & - & - & - & \\
\hline Latarjet \& Ruiz Liard & - & - & - & \\
\hline
\end{tabular}

Tabla III.- Comparación de los términos establecidos por la TAI y los términos utilizados por la literatura científica, para las arterias, venas y nervios de la ATM.

\begin{tabular}{|c|c|c|c|}
\hline TAI-Código & TAI-Términos en latín & TAI-Términos en español & $\begin{array}{l}\text { Términos en español, encontrados en } \\
\text { la literatura, que no cumplen con TAI }\end{array}$ \\
\hline A12.2.05.001 & A. CAROTIS EXTERNA & ARTERIA CARÓTIDA EXTERNA & \\
\hline A 12.2 .05 .010 & A. pharyngea ascendens & A. faríngea ascendente & A. faríngea superior \\
\hline A12.2.05.021 & A. palatina ascendens & A. palatina ascendente & \\
\hline A12.2.05.037 & A. auricularis posterior & A. auricular posterior & \\
\hline A 12.2 .05 .044 & $R$. parotideus & Rama parotídea & Ramas parotídeas \\
\hline A12.2.05.045 & A. temporalis superficialis & Arteria temporal s uperficial & \\
\hline A12.2.05.053 & A. maxillaris & Arteria maxilar & A. maxilar in terna \\
\hline A12.2.05.054 & A. auricularis profunda & A. Auricul ar profunda & \\
\hline A12.2.05.055 & A. tympanica anterior & A. timpáni ca anterior & \\
\hline A 12.2 .05 .056 & A. alveolaris inferior & A. alveolar inferior & \\
\hline A12.2.05.061 & A. meningea media & A. meníngea media & \\
\hline A12.2.05.070 & A. masseterica & A. maseterina & A. maseterica \\
\hline A 12.2 .05 .072 & A. temporalis profunda posterior & A. temporal profunda posterior & A. temporal posterior \\
\hline \multirow[t]{2}{*}{ A 12.2 .05 .073} & $R r$. pterygoidei & Rr. pterigoideas & A. pterigoidea lateral \\
\hline & & & $\begin{array}{l}\text { A. temp oral profunda media (tér mino no } \\
\text { incluido en TAI) }\end{array}$ \\
\hline A12.3.05.031 & $V$. retromandibularis & Vena retrom andibular & \\
\hline A12.3.05.032 & Vv. temporales superficiales & Venas temporal es superficiales & Vena temporal superficial \\
\hline A12.3.05.035 & Vv. maxillares & Venas maxilares & \\
\hline \multirow[t]{2}{*}{ A 12.3 .05 .036} & Plexus pterygoideus & Plexo pterigoideo & \\
\hline & & & $\begin{array}{l}\text { Plexo maxilar (término no incluido en } \\
\text { TAI) }\end{array}$ \\
\hline A14.2.01.064 & N. mandibularis & Nervio mandibular & \\
\hline A 14.2 .01 .070 & N. massetericus & N. maseterino & N. masetérico, N. masétero \\
\hline A 14.2 .01 .071 & Nn. temporales profundi & Nervios temporales profundos & N. temporal profundo \\
\hline A14.2.01.072 & N. pterygoideus lateralis & N. del músculo pterigoi deo lateral & N. pterigoideo \\
\hline A14.2.01.074 & N. auriculotemporalis & N. auriculot emporal & \\
\hline
\end{tabular}




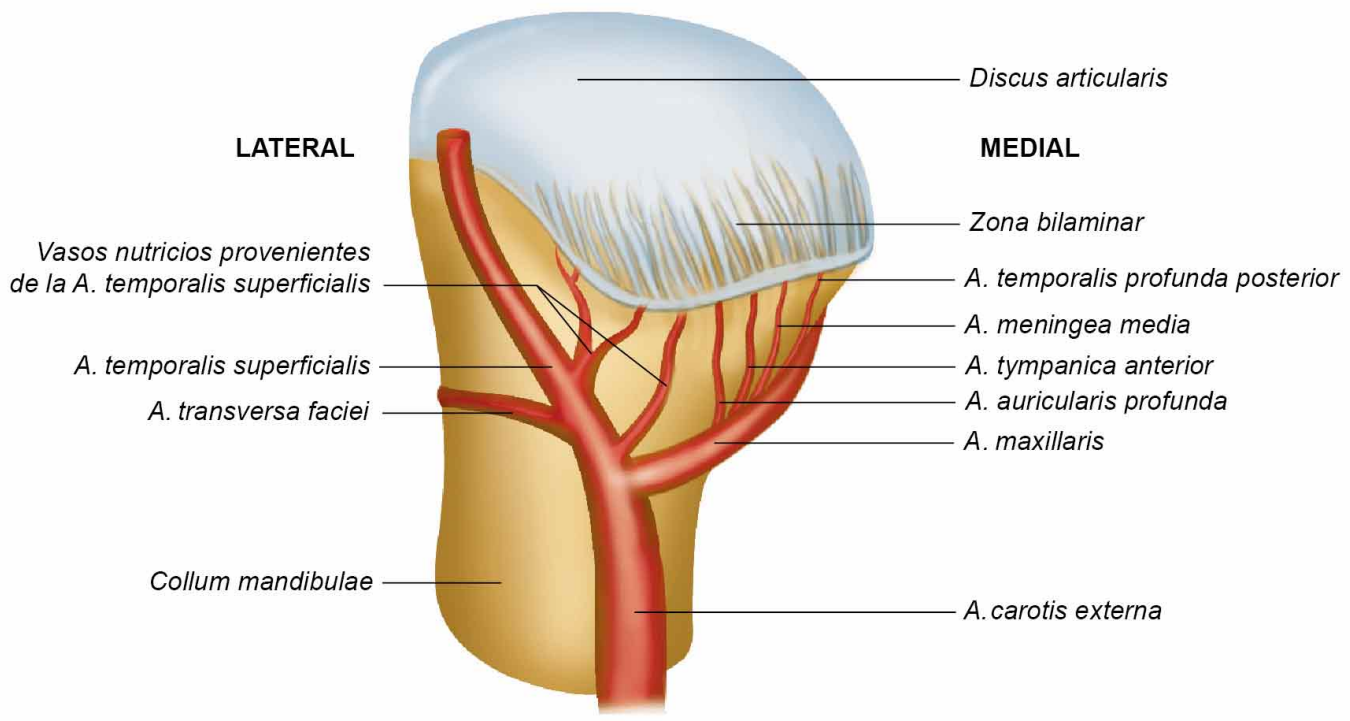

Fig. 2. Vista posterior del cóndilo de la mandíbula, con las arterias comprometidas en la irrigación del cóndilo, disco articular y zona bilaminar de la ATM.

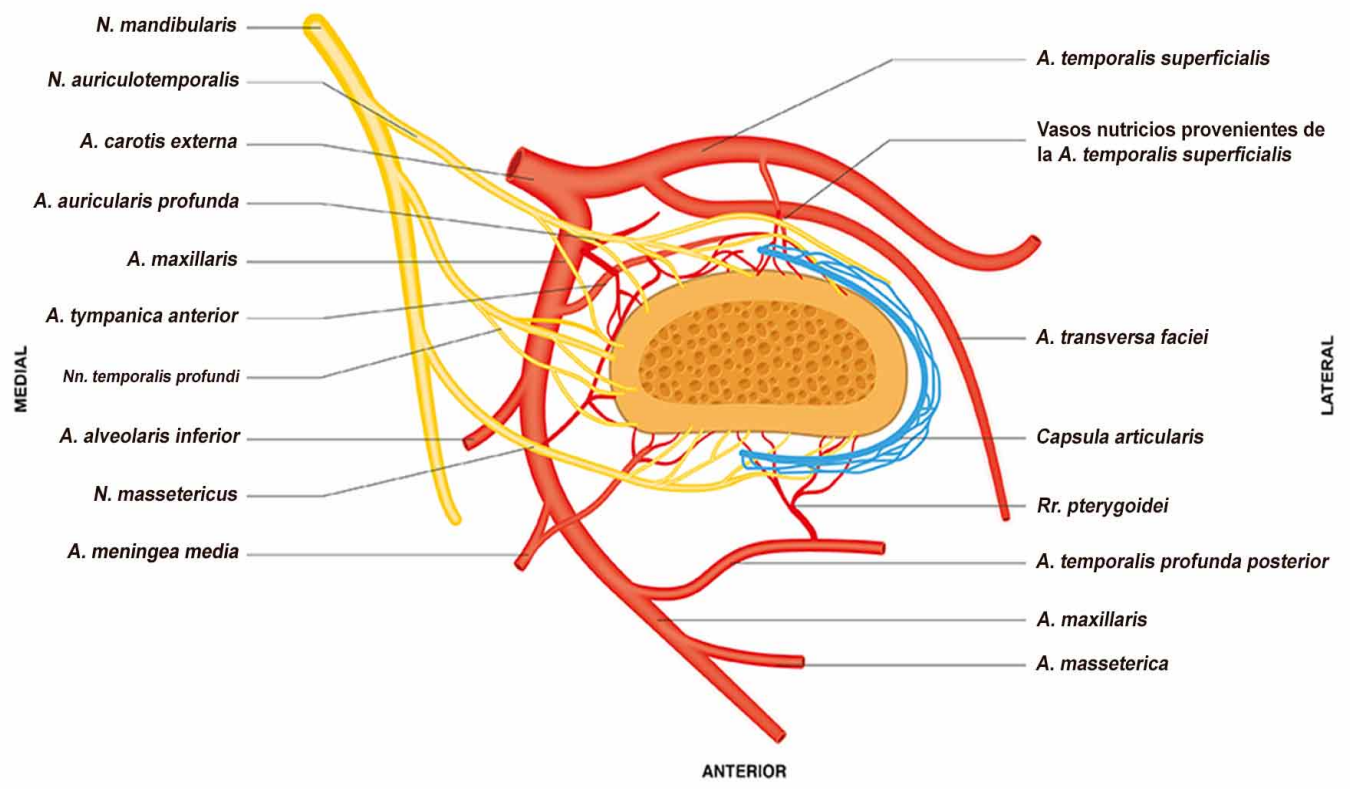

Fig. 3. Vista superior de un corte transversal del cóndilo de la mandíbula. Se observa los vasos arteriales y nervios que, a través de la cápsula articular, dan irrigación e inervación al cóndilo.

\section{DISCUSIÓN}

Irrigación arterial. Vasos comprometidos: La mayoría de los autores señalan que la irrigación de la ATM se lleva a cabo por ramas de la arteria temporal superficial y ramas de la arteria maxilar (Velayos \& Santana; Sicher \& Dubrul; Maciel; Pertes \& Gross; Bumman \& Lotzmann; Okeson;
Latarjet \& Ruiz Liard), que a su vez son ramas de la arteria carótida externa. La arteria temporal superficial irrigaría la ATM desde posterior (Sicher \& Dubrul) y la arteria maxilar desde posterior (Sicher \& Dubrul) e inferior (Okeson). Adicionalmente, la irrigación de la ATM estaría 


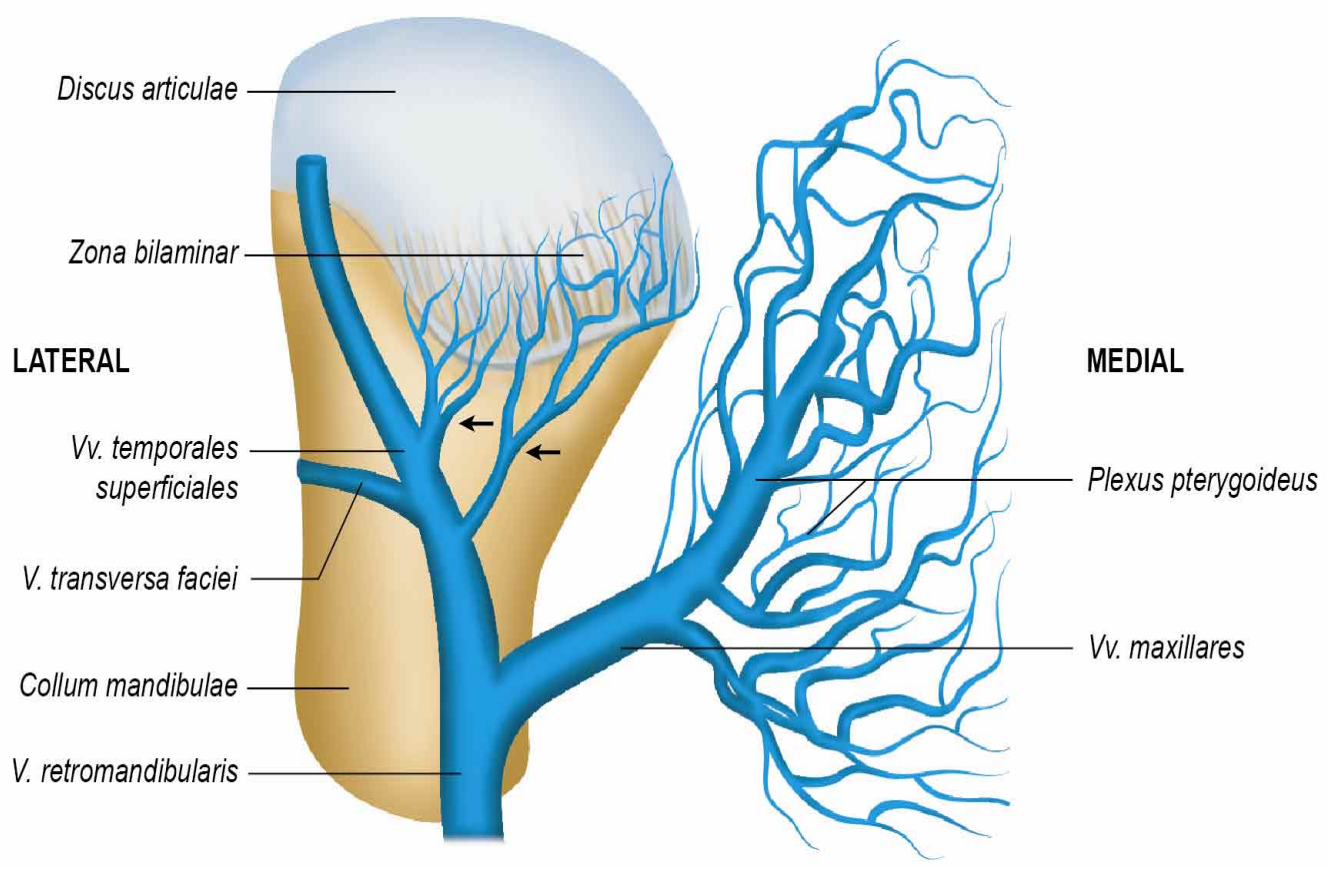

Fig. 4. Vista posterior del cóndilo de la mandíbula. Se observan las venas que drenan la zona de la articulación temporomandibular. Flechas negras: venas que drenan de la capsula articular a las venas temporales superficiales.

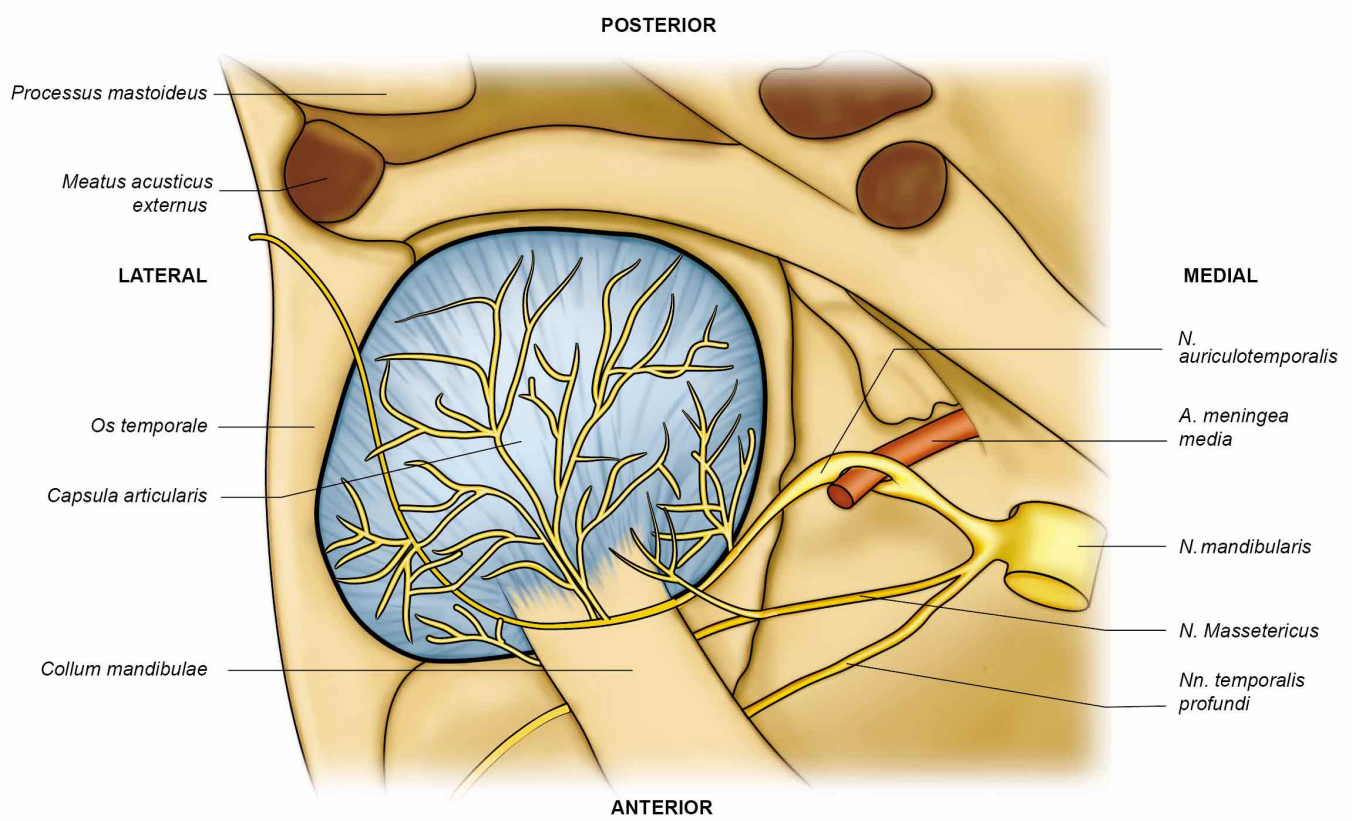

Fig. 5.- Vista inferior de la ATM izquierda. Se observa los nervios que, tras ingresar a través de la capsula articular, dan inervación a la ATM.

complementada por ramas directas de la arteria carótida externa, detalladas en la Tabla I (Figs. 1, 2 y 3 ).

También se describen la arteria timpánica anterior (Velayos \& Santana; Okeson), arteria meníngea media
(Velayos \& Santana; Maciel; Okeson; Figún \& Garino), arteria auricular profunda (Maciel; Okeson), arteria maseterina (Sicher \& Dubrul; Maciel) y la arteria temporal profunda (Velayos \& Santana) todas ramas de la arteria maxilar. Okeson señala que la arteria meníngea media irriga la ATM 


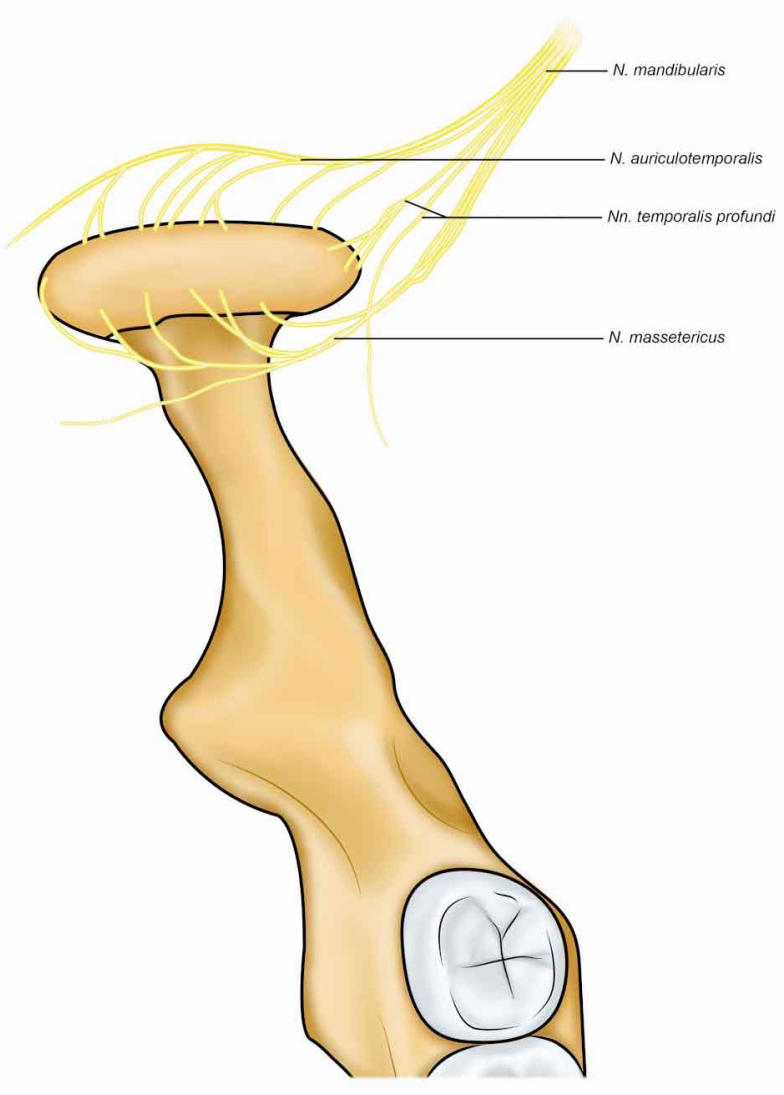

Fig. 6.- Vista anterosuperior del cóndilo de la mandíbula. Se observa los nervios temporales profundos, auriculotemporal y maseterino que dan inervación a la ATM.

por anterior y, por el contrario, Maciel indica que la irriga por posterior y lateral. La arteria maseterina también irrigaría por anterior (Sicher \& Dubrul; Maciel). Por su parte, la arteria timpánica anterior, que en su origen tiene relación con el nervio auriculotemporal, cruza la zona retroarticular y una de sus ramas irriga la zona posterior de la capsula articular y la zona bilaminar, contribuyendo a la vascularización de la articulación, según estudios realizados en ATM humanas (Mérida-Velasco et al., 1997; MéridaVelasco et al., 1999).

Cuccia et al. (2013) en un estudio realizado en 10 pacientes a los cuales se les realizó una tomografía computarizada con medio de contraste, observaron que las arterias carótida externa, temporal superficial, maxilar, alveolar inferior y meníngea media fueron observadas en el $100 \%$ de los casos. Las arterias transversa de la cara, maseterina, timpánica anterior y los ramos condilares de la arteria temporal superficial se observaron en muchos, pero no en todos los casos (70\%, $60 \%, 60 \%$ y $50 \%$, respectivamente). Un estudio angiográfico similar fue realizado por Takagi et al. (1998) en 10 pacientes adultos asintomáticos donde la arteria carótida externa fue detectada en el $100 \%$ de los casos, y las arterias maxilar, temporal superficial, transversa de la cara, timpánica anterior y auricular profunda en menor medida $(80 \%, 90 \%, 75 \%, 25 \%$ y $5 \%$, respectivamente). Así, las arterias con diámetro fino son menos observadas. Esto podría explicar que existan diferencias entre los autores al momento de reportar los vasos comprometidos en la irrigación de la ATM (Tabla I).

\section{Irrigación arterial según estructura anatómica}

\section{Cápsula}

Sicher \& Dubrul señalan que todos los vasos que pueden ser identificados en un radio de $3 \mathrm{~cm}$ de la ATM dan ramificaciones para la capsula. Estos vasos arteriales se encuentran mencionados en la Tabla I. La capsula esta irrigada por vasos que provienen de todas las direcciones y que constituyen el plexo de la capsula, que luego irrigaría el resto de la ATM (Boyer et al., 1964). La zona posterior y medial de la capsula están muy irrigadas y en menor medida la zona anterior y el ligamento lateral (Boyer et al.)

\section{Proceso condilar}

Para Cuccia et al. es claro que la mayor parte de la irrigación se obtiene de la parte lateral y medial del cóndilo. Esto es confirmado por Takagi et al. (1994) quienes reportan que el aporte vascular de la articulación parece provenir de la cara medial y lateral del cóndilo y de los aspectos anteriores y posteriores del disco articular. Por el contrario, Boyer et al. describen a la zona posterior del cóndilo como la más vascularizada.

Los vasos sanguíneos (mencionados en la Tabla I) irrigan primeramente a la cápsula articular, posteriormente ingresan a través de ella e irrigan el resto de las estructuras de la ATM, entre ellos el cóndilo. Adicionalmente, el cóndilo recibe irrigación por los vasos nutricios provenientes de la arteria alveolar inferior, que tras pasar por el foramen mandibular otorga ramas que viajan a través de los espacios medulares de la rama y cuello hacia el cóndilo (Fig. 1) (Okeson; Funakoshi, 2000). Gracias a esto la necrosis avascular del cóndilo ocurre en pocas ocasiones (Bumann \& Lotzmann).

\section{Disco articular}

La periferia del disco está bien vascularizada, aunque no uniformemente (Boyer et al.). Los vasos se ramifican a medida que avanzan hacia el centro del disco y terminan en una zona bien delimitada, circunscribiendo la zona central relativamente avascularizada (Cuccia et al.; Boyer et al.). 
La porción posterior del disco está ampliamente vascularizada (Cuccia et al.; Boyer et al.). Esta zona está irrigada por la arteria temporal superficial y por ramas de la arteria maxilar (arteria timpánica anterior y auricular profunda) (Siéssere et al., 2004).

Según Cuccia et al. la región anterior está relativamente desprovista de vasos mientras que para Boyer et al. la zona anterior del disco está ricamente irrigada y su plexo arterial se continúa con la irrigación del musculo pterigoideo lateral, que se inserta en el sector anterior del disco.

\section{Fosa articular}

El área funcional de la fosa es avascular. Existe una zona de delimitación vascular donde la zona posterior es la más vascular y parte más profunda es avascular. El lecho vascular termina justo antes de la convexidad mayor del tubérculo articular (Boyer et al.)

Drenaje venoso: El drenaje venoso de la ATM se encuentra bastante menos mencionado y descrito en la literatura. Sicher \& Dubrul señalan que el drenaje venoso es más difuso que la irrigación arterial, y que forma un plexo abundante en toda la circunvalación de la capsula y en la zona retrodiscal (Fig. 4). Mérida-Velasco et al. analizaron 45 fetos humanos y observaron numerosos espacios venosos en la zona retroarticular, constituyendo el plexo venoso retrodiscal. Los abundantes vasos venoso de la zona retrodiscal se llenan o vacían de acuerdo al movimiento condilar (Velayos \& Santana). En la zona anterior también se observa drenaje venoso pero en menor grado (Sicher \& Dubrul).

Según la literatura consultada el drenaje venoso de la ATM estaría dado por las venas temporales superficiales (Bumann \& Lotzmann) y por el plexo pterigoideo (Bumman \& Lotzmann; Velayos \& Santana), que drena a las venas maxilares (Fig. 4). Las venas temporales superficiales y las venas maxilares drenan a la arteria retromandibular (Schunke et al.) (Fig. 4). Bumman \& Lotzmann mencionan un plexo maxilar, que aportaría en el drenaje de la ATM, pero que no está descrito por ningún otro autor de la literatura consultada y que no se encuentra en la TAI.

Inervación sensitiva: La inervación sensitiva de la ATM está dada por el nervio mandibular, ramo del V par craneal (Velayos \& Santana; Sicher \& Dubrul; Maciel; Pertes \& Gross; Okeson; Latarjet \& Ruiz Liard) (Figs. 5 y 6).

El nervio mandibular otorga los ramos auriculotemporal y maseterino (Velayos \& Santana; Sicher \& Dubrul; Maciel; Pertes \& Gross; Okeson; Latarjet \& Ruiz; Bumann \& Lotzmann; Martínez-Ross; Figun \& Garino;
Schunke et al.), y los nervios temporales profundos (Maciel; Pertes \& Gross; Okeson; Latarjet \& Ruiz; Martinez-Ross; Schunke et al.) (Figs. 5 y 6) y nervio del músculo pterigoideo lateral (Maciel).

El nervio maseterino inerva la parte anterior y medial de la capsula y la ATM y los nervios temporales profundos inervan la zona anterolateral de la capsula y la ATM (Sicher \& Dubrul; Martínez-Ross). El nervio auriculotemporal da inervación sensitiva a la porción medial, lateral (Sicher \& Dubrul) y posterior de la ATM (Sicher \& Dubrul; Okeson; Martínez-Ross) (Figs. 5 y 6 )

Cuatro terminaciones nerviosas se encuentran en toda la capsula, el ligamento lateral y la zona bilaminar y son responsables de la propiocepción. Los mecanoreceptores de Ruffini (tipo I), entregan información de la postura e inhiben los reflejos de los músculos inhibidores. Los corpúsculos de Pacini (tipo II), conducen información del movimiento. El órgano tendinoso de Golgi (tipo III) es también un mecanorreceptor que se activa cuando la mandíbula alcanza la mayor amplitud de sus movimientos, y las terminaciones libres (tipo IV), son las responsables de la percepción del dolor (Bumann \& Lotzmann).

Asaki et al. (2006) analizaron 22 ATM mediante microscopía óptica y electrónica, y observaron terminaciones nerviosas sensitivas en el sector anterior y posterior del disco y en la zona de transición de la membrana sinovial a la capsula, en el sector posterior. Wink et al. (1992) reportaron que terminaciones nerviosas tipo I, II y III y nervios ligeramente mielinizados fueron identificados en el tejido conectivo pericapsular y en el disco y que la densidad de los elementos neuronales es mayor en la periferia del disco y progresivamente decrece hacia el centro, que esta desprovisto de ellas. La concentración de elementos nerviosos parece ser mayor en la zona posterior del disco, y en menor medida en la zona anterior (Wink et al.).

Inervación simpática: Según Bumann \& Lotzmann las neuronas simpáticas se encuentran mayoritariamente en la porción articular posterior. Las neuronas simpáticas permiten el control vasomotor y juegan un rol en la percepción del dolor. El control vasomotor permite la regulación óptima del volumen sanguíneo durante los movimientos condilares excursivos e incursivos (Bumman \& Lotzmann).

La relación entre las fibras simpáticas y sensoriales en la ATM es de 3:1 aproximadamente (Bumann \& Lotzmann). Figún \& Garino señalan que la capsula, los ligamentos y la membrana sinovial son tejidos muy ricos en fibras nerviosas, especialmente de origen simpático, por lo que sus reacciones inflamatorias son muy vivas. 


\section{CONCLUSIÓN}

Los autores concuerdan en los principales vasos (arteria maxilar y la arteria temporal superficial) y ramos nerviosos (auriculotemporal y maseterino) que dan irrigación e inervación a la ATM, aunque también se mencionan otros vasos y nervios menos descritos. Por su parte, el drenaje venoso de la ATM se encuentra escasamente descrito en la literatura. Faltan artículos científicos basados en el análisis a cadáveres humanos orientados a describir la irrigación otorgada por vasos de calibre fino y el drenaje venoso. Por lo general, los términos usados en la literatura para referirse a los componentes vasculares y nerviosos de la ATM cumplen con los términos establecidos por la Terminologia Anatomica Internacional.

FUENTES, R.; OTTONE, N. E.; SARAVIA, D. \& BUCCHI, C. Irrigation and innervation of the temporomandibular joint. A literature review. Int. J. Morphol., 34(3):1024-1033, 2016.

SUMMARY: The knowledge of the afferent nerves, vascular supply and venous drainage of the temporomandibular joint is relevant to understand the physiology of the stomatognathic system. The aim of this study was to identify the blood vessels and nerves involved in the blood supply and innervation of the TMJ, and if the terms used in the scientific literature respects the Terminologia Anatomica. Ten books and 11 scientific articles were included. A literature review was conducted. Most authors suggest that TMJ irrigation is carried out by branches of the superficial temporal artery and branches of the maxillary artery, which are branches of the external carotid artery. The venous drainage would be given by the superficial temporal vein and by the pterygoid plexus. The sensory innervation was given by the mandibular nerve, branch of the $\mathrm{V}$ cranial nerve and its branches auriculotemporal, masseteric and the deep temporal nerves. The authors agree on the major vessels and nerve branches that provide irrigation and innervation to the TMJ, although other less described vessels and nerves are also mentioned. The venous drainage of the TMJ is poorly described in the literature.

KEY WORDS: Temporomandibular joint; Irrigation; Innervation; Venous drainage.

\section{REFERENCIAS BIBLIOGRÁFICAS}

Asaki, S.; Sekikawa, M. \& Kim, Y. Sensory innervation of temporomandibular joint disk. J. Orthop. Surg. (Hong Kong), 14(1):3-8, 2006.

Boyer, C. C.; Williams, T. W. \& Stevens, F. H. Blood supply of the temporomandibular joint. J. Dent. Res., 43:224-8, 1964.

Bumann, A. \& Lotzmann, U. Atlas de Diagnóstico Funcional y Principios Terapéuticos en Odontología. Barcelona, Masson, 2000.

Colbert, S.; Cameron, M. \& Williams, J. Septic thrombosis of the cavernous sinus and dental infection. Br. J. Oral Maxillofac. Surg., 49(6):e25-6, 2011.

Cuccia, A. M.; Caradonna, C.; Caradonna, D.; Anastasi, G.; Milardi, D.; Favaloro, A.; De Pietro, A.; Angileri, T. M.; Caradonna, L. \& Cutroneo, G. The arterial blood supply of the temporomandibular joint: an anatomical study and clinical implications. Imaging Sci. Dent., 43(1):37-44, 2013.

Ebright, J. R.; Pace, M. T. \& Niazi, A. F. Septic thrombosis of the cavernous sinuses. Arch. Intern. Med., 161(22):2671-6, 2001.
Figún, M. \& Garino, R. Anatomía Odontológica Funcional y Aplicada. Buenos Aires, El Ateneo, 2002.

Funakoshi, K. Nutrient arteries of the temporomandibular joint: an anatomical and a pathological study. Okajimas Folia Anat. Jpn., 78(1):7-16, 2001.

Latarjet, M. \& Ruiz Liard, A. Anatomía Humana. Tomo 2. $2^{\mathrm{a}}$ ed. Buenos Aires, Editorial Médica Panamericana, 2005.

Maciel, R.N. ATM e Dores Craneofaçiais. Fisiopatologia Básica. São Paulo, Santos, 2003.

Martínez-Ross, E. Disfunción Témporomandibular. Santa Fé de Bogotá, Ediciones Monserrate, 1992.

Mérida-Velasco, J. R.; Rodríguez-Vázquez, J. F. \& Jiménez-Collado, J. Anterior tympanic artery: course, ramification and relationship with the temporomandibular joint. Acta Anat. (Basel), 158(3):222-6, 1997.

Mérida-Velasco, J. R.; Rodríguez-Vázquez, J. F.; Mérida-Velasco, J. A. \& Jiménez-Collado, J. The vascular relationship between the temporomandibular joint and the middle ear in the human fetus. J. Oral Maxillofac. Surg., 57(2):146-53, 1999. 
Okeson, J. Tratamiento de Oclusión y Afecciones Temporomandibulares. $5^{\text {a }}$ ed. Madrid, Mosby Inc., 2003.

Pertes, R. \& Gross, S. Tratamento Clínico das Disfunçoes Temporomandibulares e da Dor Orofacial. São Paulo, Quintessence, 2005.

Rubiano, C. M. Tratamiento con Placas y Corrección Oclusal por Tallado Selectivo. Bogotá, Amolca, 2005.

Schunke, M.; Schulte, E. \& Schumacher, U. Prometheus. Texto y Atlas de Anatomía. Tomo 3. $2^{\mathrm{a}}$ ed. Buenos Aires, Editorial Médica Panamericana, 2010.

Sicher, H. \& Dubrul, E. Anatomia Oral. $8^{\mathrm{a}}$ ed. São Paulo, Artes Medicas, 1991.

Siéssere, S.; Vitti, M.; Sousa, L. G.; Semprini, M. \& Regalo, S. C. H. Bilaminar zone: Anatomical aspects, irrigation, and innervation. Braz. J. Morphol. Sci., 21(4):217-20, 2004.

Takagi, R.; Shimoda, T.; Westesson, P. L.; Takahashi, A.; Morris, T. W.; Sano, T. \& Moses, J. J. Angiography of the temporomandibular joint. Description of an experimental technique with initial results. Oral Surg. Oral Med. Oral Pathol., 78(4):539-43, 1994.

Takagi, R.; Westesson, P. L.; Ohashi, Y. \& Togashi, H. MR angiography of the TMJ in symptomatic volunteers. Oral Radiol., 14(2):69-74, 1998.

Vasconcellos, A. H.; Sousa, E. M. A. \& Cavalcante, M. L. T. M. H. Clasificación de la articulación Témporomandibular: Aspectos anátomofuncionales. Int. J. Odontostomat., 1(1):25-8, 2007.

Velayos, J. L. \& Santana H. D. Anatomía de la Cabeza con Enfoque Odontoestomatológico. $3^{\mathrm{a}} \mathrm{ed}$. Madrid, Editorial Médica Panamericana, 2001.

Wink, C. S.; Onge, S.T., M. \& Zimny, M. L. Neural elements in the human temporomandibular articular disc. J. Oral Maxillofac. Surg., 50(4):334-7, 1992.

\author{
Dirección de correspondencia \\ Dr. Ramón Fuentes \\ $\mathrm{CICO}$ - Centro de Investigación en Ciencias \\ Odontológicas \\ Facultad de Odontología \\ Universidad de La Frontera \\ Manuel Montt 112, Casilla 54-D \\ Temuco \\ CHILE
}

Email: ramon.fuentes@ufrontera.cl 